Comment. Math. Helv. 73 (1998) 237-258

(C) 1998 Birkhäuser Verlag, Basel

\title{
The double Coxeter arrangement
}

\author{
Louis Solomon and Hiroaki Terao*
}

\begin{abstract}
Let $V$ be Euclidean space. Let $W \subset \mathbf{G L}(V)$ be a finite irreducible reflection group. Let $\mathcal{A}$ be the corresponding Coxeter arrangement. Let $S$ be the algebra of polynomial functions on $V$. For $H \in \mathcal{A}$ choose $\alpha_{H} \in V^{*}$ such that $H=\operatorname{ker}\left(\alpha_{H}\right)$. The arrangement $\mathcal{A}$ is known to be free: the derivation module $D(\mathcal{A})=\left\{\theta \in \operatorname{Der}_{S} \mid \theta\left(\alpha_{H}\right) \in S \alpha_{H}\right\}$ is a free $S$-module with generators of degrees equal to the exponents of $W$. In this paper we prove an analogous theorem for the submodule $E(\mathcal{A})$ of $D(\mathcal{A})$ defined by $E(\mathcal{A})=\left\{\theta \in \operatorname{Der}_{S} \mid \theta\left(\alpha_{H}\right) \in S \alpha_{H}^{2}\right\}$. The degrees of the basis elements are all equal to the Coxeter number. The module $E(\mathcal{A})$ may be considered a deformation of the derivation module for the Shi arrangement, which is conjectured to be free. The proof is by explicit construction using a derivation introduced by K. Saito in his theory of flat generators.
\end{abstract}

Mathematics Subject Classification (1991). Primary 52B30; secondary 05E15.

Keywords. Hyperplane arrangement, free arrangement, Shi arrangement, reflection group, basic invariants, Coxeter number, Jacobian matrix.

\section{$\S$ 1. Introduction}

Let $V$ be a Euclidean space of dimension $l$ over $\mathbf{R}$. Let $($,$) denote the positive$ definite symmetric bilinear form on $V$. Let $W \subseteq \mathbf{G L}(V)$ be a finite group generated by orthogonal reflections [Bou, V.2.3]. Let $\mathcal{A}$ be the corresponding Coxeter arrangement, the set of hyperplanes $H \subset V$ such that $W$ contains the orthogonal reflection which fixes $H$. Let $S$ be the algebra of polynomial functions on $V$. The algebra $S$ is naturally graded by $S=\bigoplus_{q \geq 0} S_{q}$ where $S_{q}$ is the space of homogeneous polynomials of degree $q$. Thus $S_{1}=V^{*}$ is the dual space of $V$. Let $\operatorname{Der}_{S}$ be the $S$-module of $\mathbf{R}$-derivations of $S$. We say that $\theta \in \operatorname{Der}_{S}$ is homogeneous of degree $q$ if $\theta\left(S_{1}\right) \subseteq S_{q}$. Choose for each hyperplane $H \in \mathcal{A}$ a linear form $\alpha_{H} \in V^{*}$ such that $H=\operatorname{ker}\left(\alpha_{H}\right)$. Define $Q \in S$ by

$$
Q=\prod_{H \in \mathcal{A}} \alpha_{H}
$$

*Second author partially supported by NSF Grant DMS9504457. 
The polynomial $Q$ is uniquely determined, up to a constant multiple, by the group $W$. Let

$$
D(\mathcal{A})=\left\{\theta \in \operatorname{Der}_{S} \mid \theta\left(\alpha_{H}\right) \in S \alpha_{H}\right\} .
$$

K. Saito [Sai1, Theorem], [Ter,Theorem 2] proved that $D(\mathcal{A})$ is a free $S$-module of rank $l$ and that a set of basis elements for $D(\mathcal{A})$ as $S$-module may be described as follows. Let $R=S^{W}$ be the algebra of $W$-invariant polynomials on $V$. By a theorem of Shephard, Todd, and Chevalley [Bou, V.5.3, Theorem 3] there exist algebraically independent homogeneous polynomials $f_{1}, \ldots, f_{l} \in R$ such that $R=$ $\mathbf{R}\left[f_{1}, \ldots, f_{l}\right]$. Let $x_{1}, \ldots, x_{l}$ be an orthonormal basis for $V^{*}$. Let $\partial_{i}$ be partial differentiation with respect to $x_{i}$. Define $\theta_{j} \in \operatorname{Der}_{S}$ by $\theta_{j}=\sum_{i=1}^{l}\left(\partial_{i} f_{j}\right) \partial_{i}$ for $1 \leq j \leq l$. Then $\left\{\theta_{1}, \ldots, \theta_{l}\right\}$ is an $S$-basis for $D(\mathcal{A})$. Note that $\theta_{j}$ is homogeneous of degree $\operatorname{deg}\left(f_{j}\right)-1$. The integers $m_{j}=\operatorname{deg}\left(f_{j}\right)-1$ for $1 \leq j \leq l$ are the exponents of $W$ [Bou, V.6.2, Proposition 3].

In this paper we will prove an analogous theorem for the submodule $E(\mathcal{A})$ of $D(\mathcal{A})$ defined by

$$
E(\mathcal{A})=\left\{\theta \in \operatorname{Der}_{S} \mid \theta\left(\alpha_{H}\right) \in S \alpha_{H}^{2}\right\} .
$$

Note that we have replaced $S \alpha_{H}$ in (1.2) by $S \alpha_{H}^{2}$ in (1.3), which explains the phrase "double Coxeter arrangement" in the title of this paper. If $\theta \in D(\mathcal{A})$ and $\alpha=\alpha_{H}$ then $\theta(Q)=\theta(\alpha \cdot Q / \alpha)=(Q / \alpha) \theta(\alpha)+\alpha \theta(Q / \alpha) \in S \alpha$ so that $\theta(Q) \in S Q$. On the other hand, it may happen that $\theta \in E(\mathcal{A})$, but $\theta(Q) \notin S Q^{2}$.

To state our theorem we need some preliminary definitions. Assume that $W$ is an irreducible subgroup of $\mathbf{G L}(V)$. The form $($,$) on V$ induces a positive definite symmetric bilinear form on $V^{*}$, sometimes called the inverse form, which we also write as $($,$) . Let e_{1}, e_{2}, \ldots, e_{l}$ be a basis for $V$. We do not assume that $e_{1}, e_{2}, \ldots, e_{l}$ is an orthonormal basis unless orthonormality is explicitly stated. Let $x_{1}, x_{2}, \ldots, x_{l}$ be the dual basis for $V^{*}$. Let $\Gamma$ be the matrix of the inverse form with respect to the chosen basis $x_{1}, \ldots, x_{l}$. Thus $\Gamma_{i j}=\left(x_{i}, x_{j}\right)$. Number the invariant polynomials $f_{j}$ so that $\operatorname{deg}\left(f_{1}\right) \leq \cdots \leq \operatorname{deg}\left(f_{l}\right)$. Since $W$ is irreducible, the Coxeter number $h$ of $W$ is defined [Bou, V.6.1] and $h=\operatorname{deg}\left(f_{l}\right)$ [Bou, V.6.2]. Let $K$ be the quotient field of $S$. K. Saito [Sai2, 2.2], [SYS, (1.6)] studied an Rderivation $D \in \operatorname{Der}_{K}$ such that $D f_{j}=0$ for $1 \leq j \leq l-1$ and $D f_{l} \in \mathbf{R}^{*}$. This derivation is uniquely determined, up to a constant multiple, by the group $W$ and does not depend on choice of basic invariants $f_{1}, \ldots, f_{l}$. Define rational fuctions $h_{j} \in K$ for $1 \leq j \leq l$ by

$$
h_{j}=D x_{j} .
$$

Let $J\left(h_{1}, \ldots, h_{l}\right)$ be the Jacobian matrix, labeled so that $\partial_{i} h_{j}$ is its $(i, j)$ entry. We will prove in Corollary 3.32 that $J\left(h_{1}, \ldots, h_{l}\right)$ is invertible over $K$. This is perhaps the most difficult point in the paper. Furthermore $J\left(h_{1}, \ldots, h_{l}\right)^{-1}$ has entries in $S$. The structure of the $S$-module $E(\mathcal{A})$ is given by the following theorem.

Theorem 1.4. Let $W \subseteq \mathbf{G L}(V)$ be a finite irreducible group generated by reflec- 
tions. Define an $l \times l$ matrix $P$ by

$$
P=\Gamma J\left(h_{1}, \ldots, h_{l}\right)^{-1} .
$$

Define $\xi_{1}, \ldots, \xi_{l} \in \operatorname{Der}_{S}$ by $\xi_{j}=\sum_{i=1}^{l} p_{i j} \partial_{i}$ for $1 \leq j \leq l$, where $p_{i j}$ is the $(i, j)$ entry of $P$. Then $\xi_{i} \in E(\mathcal{A})$, and $E(\mathcal{A})$ is a free $S$-module with basis $\xi_{1}, \ldots, \xi_{l}$.

Note that if $x_{1}, \ldots, x_{l}$ is an orthonormal basis for $V^{*}$ then $\Gamma$ is the identity matrix and (1.5) becomes $P=J\left(h_{1}, \ldots, h_{l}\right)^{-1}$. We will see that all entries of $P$ are homogeneous of degree equal to the Coxeter number $h$. Thus all derivations $\xi_{1}, \ldots, \xi_{l}$ are homogeneous of degree $h$. We will prove in Proposition 4.7 that the homogeneous component $E(\mathcal{A})_{h}$ of degree $h$ is isomorphic to $V^{*}$ as $W$-module. The rational functions $h_{1}, \ldots, h_{l}$ may be computed as follows. Let $J\left(f_{1}, \ldots, f_{l}\right)$ be the Jacobian matrix of $f_{1}, \ldots, f_{l}$. Since $f_{1}, \ldots, f_{l}$ are algebraically independent, $J\left(f_{1}, \ldots, f_{l}\right)$ is invertible over $K$. Then $\left[h_{1}, \ldots, h_{l}\right]$ is, up to constant multiple, the $l$-th row of $J\left(f_{1}, \ldots, f_{l}\right)^{-1}$.

Remark 1.6. Define polynomials $u_{1}, \ldots, u_{l} \in S$ by $u_{i}=Q h_{i}$ for $1 \leq i \leq l$. Invertibility of the matrix $J\left(h_{1}, \ldots, h_{l}\right)$ is equivalent to invertibility of the matrix $J\left(u_{1}, \ldots, u_{l}\right)$, which was conjectured in [Sol2].

Remark 1.7. The definition (1.3) of $E(\mathcal{A})$ is due to Ziegler [Zie, Definition 4] who developed the theory of multiarrangements. A double Coxeter arrangement is a multiarrangement with multiplicity two for each hyperplane belonging to the Coxeter arrangement.

Remark 1.8. We were led to study the double Coxeter arrangements by an attempt to understand the Shi arrangements [Shi1], [Sshi2]. Suppose that $W$ is a Weyl group. Choose a crystallographic root system in $V^{*}$ and choose the linear forms $\alpha_{H}$ so that $\pm \alpha_{H}$ is a root for each $H \in \mathcal{A}$. Let $\alpha_{1}, \ldots, \alpha_{n} \in V^{*}$ be a system of positive roots. The Shi arrangement $\tilde{\mathcal{A}}$ of type $W$ is an affine arrangement with $2 n$ hyperplanes whose defining polynomial is $\tilde{Q}=\prod_{i=1}^{n}\left(\alpha_{i}-1\right) \prod_{i=1}^{n} \alpha_{i}$. Shi arrangements have been studied by Stanley [Sta1], [Sta2] and others. A special case of a conjecture due to Edelman and Reiner [EdR, Conjecture 3.3] states that the cone [OrT, p.14] c $\tilde{\mathcal{A}}$ of each Shi arrangement is a free arrangement with exponents $\{1, h, \ldots, h\}$ [OrT, Definition 4.15 , Definition 4.25$]$; the module $D(\mathbf{c} \tilde{\mathcal{A}})$ is a free module over $\mathbf{R}\left[x_{0}, \ldots, x_{l}\right]$. Athanasiadis [Ath] verified this conjecture for type $\mathrm{A}_{l}$. Note that the restriction (as a multiarrangement) of $\mathbf{c} \tilde{\mathcal{A}}$ to the infinite hyperplane $x_{0}=0$ is the double Coxeter arrangement. Therefore, if the conjecture is true, then, by Ziegler's theorem [Zie, Theorem 11], we may conclude that the double Coxeter arrangement is a free arrangement with exponents $\{h, h, \ldots, h\}$, which is true by our main result, Theorem 1.4. So Theorem 1.4 may be regarded as a piece of evidence supporting the conjecture.

Here is an outline of the paper. In Section 2 we introduce more notation and 
state some elementary facts. In Section 3 we prove the invertibility of $J\left(h_{1}, \ldots, h_{l}\right)$. In Section 4 we complete the proof of Theorem 1.4. In Section 5 we compute the matrix $P$ in case $l=2$ and in case $W$ has type $\mathrm{B}_{l}$. In Section 6 we use the invertibility of the matrix $J\left(u_{1}, \ldots, u_{l}\right)$ to describe the differential 1-forms which are anti-invariant under $W$.

\section{$\S 2$. Notation and preliminary definitions}

In this Section we fix more notation, state some elementary facts about derivations and differential forms, and introduce some of the main constructs in the argument. We often use the notation of Section 1 without comment. When convenient we choose a basis $e_{1}, \ldots, e_{l}$ for $V$ and let $x_{1}, \ldots, x_{l}$ denote the dual basis for $V^{*}$. Let $\langle\rangle:, V^{*} \times V \rightarrow \mathbf{R}$ denote the natural pairing. Thus $\left\langle x_{i}, e_{j}\right\rangle=\delta_{i j}$. Let $\operatorname{Der}_{S}$ be the $S$-module of $\mathbf{R}$-derivations of $S$. For each $v \in V$ let $\partial_{v} \in \operatorname{Der}_{S}$ be the unique derivation such that $\partial_{v} x=\langle x, v\rangle$ for $x \in V^{*}$. Define $\partial_{i} \in \operatorname{Der}_{S}$ by $\partial_{i}=\partial_{e_{i}}$. Then $\partial_{i} x_{j}=\delta_{i j}$ and $\operatorname{Der}_{S}$ is a free $S$-module with basis $\partial_{1}, \ldots, \partial_{l}$. There is a natural isomorphism $S \otimes V \rightarrow \operatorname{Der}_{S}$ of $S$-modules given by

$$
f \otimes v \mapsto f \partial_{v}
$$

for $f \in S$ and $v \in V$. Let $\Omega_{S}^{1}=\operatorname{Hom}_{S}\left(\operatorname{Der}_{S}, S\right)$ be the $S$-module dual to $\operatorname{Der}_{S}$. Define $d: S \rightarrow \Omega_{S}^{1}$ by $d f(\theta)=\theta(f)$ for $f \in S$ and $\theta \in \operatorname{Der}_{S}$. Then $d\left(f f^{\prime}\right)=(d f) f^{\prime}+$ $f\left(d f^{\prime}\right)$ for $f, f^{\prime} \in S$. Furthermore, $\Omega_{S}^{1}$ is a free $S$-module with basis $d x_{1}, \ldots, d x_{l}$ and $d f=\sum_{i=1}^{l}\left(\partial_{i} f\right) d x_{i}$. There is a natural isomorphism $S \otimes V^{*} \rightarrow \Omega_{S}^{1}$ of $S$ modules given by

$$
f \otimes x \mapsto f d x
$$

for $f \in S$ and $x \in V^{*}$. The modules $\operatorname{Der}_{S}$ and $\Omega_{S}^{1}$ inherit gradings from $S$ which are defined by $\operatorname{deg}\left(f \partial_{v}\right)=\operatorname{deg}(f)$ and $\operatorname{deg}(f d x)=\operatorname{deg}(f)$ if $f \in S$ is homogeneous.

We define several $W$-module structures which stem from the given $W$-module structure on $V$. If $f \in S$ define $w f \in S$ by $(w f)(v)=f\left(w^{-1} v\right)$ for $v \in V$. This makes $S$ a $W$-module and $W$ acts as a group of $\mathbf{R}$-algebra automorphisms of $S$. In particular $V^{*}=S_{1}$ has a $W$-module structure, and $\langle w x, w v\rangle=\langle x, v\rangle$ for $w \in W$, $x \in V^{*}$ and $v \in V$. The spaces $S \otimes V$ and $S \otimes V^{*}$ have $W$-module structures given by $w(f \otimes v)=w f \otimes w v$ and $w(f \otimes x)=w f \otimes w x$. We give $\operatorname{Der}_{S}$ a $W$ module structure by defining $(w \theta)(f)=w\left(\theta\left(w^{-1} f\right)\right)$ for $w \in W, \theta \in \operatorname{Der}_{S}$ and $f \in S$. Then $w \partial_{v}=\partial_{w v}$ for $w \in W$ and $v \in V$. To check this it suffices to check that both derivations $w \partial_{v}$ and $\partial_{w v}$ have the same effect on $V^{*}$. This is so since $\left(w \partial_{v}\right)(x)=\partial_{v}\left(w^{-1} x\right)=\left\langle w^{-1} x, v\right\rangle=\langle x, w v\rangle=\partial_{w v}(x)$. We give $\Omega_{S}^{1}$ a $W$-module structure by defining $w(f d x)=(w f) d(w x)$ for $w \in W, f \in S$ and $x \in V^{*}$. In particular, $w(d x)=d(w x)$. The isomorphisms in (2.1) and (2.2) are $W$-module isomorphisms.

Define an $S$-bilinear form $():, \Omega_{S}^{1} \times \Omega_{S}^{1} \rightarrow S$ by 


$$
\left(f d x, f^{\prime} d x^{\prime}\right)=f f^{\prime}\left(x, x^{\prime}\right)
$$

for $f, f^{\prime} \in S$ and $x, x^{\prime} \in V^{*}$ where $\left(x, x^{\prime}\right)$ denotes the form on $V^{*}$ inverse to the given form on $V$. In particular, $\left(d x, d x^{\prime}\right)=\left(x, x^{\prime}\right)$ for $x, x^{\prime} \in V^{*}$. If $w \in W$ then, since $\left(w x, w x^{\prime}\right)=\left(x, x^{\prime}\right)$ for $w \in W$, it follows from $(2.3)$ that $w\left(\omega, \omega^{\prime}\right)=\left(w \omega, w \omega^{\prime}\right)$ for $\omega, \omega^{\prime} \in \Omega_{S}^{1}$.

Let $K$ be the quotient field of $S$. We make various conventions about matrices over $K$ which will be used throughout the paper. Let $\mathbf{M}_{l}(K)$ denote the set of $l \times l$ matrices over $K$. We use similar notation for matrices over other rings. If $A$ is any rectangular matrix over $K$ we let $A_{i j}$ denote the $(i, j)$ entry of $A$ and let $A^{\top}$ denote the transpose of $A$. It is sometimes convenient to define a matrix as $A=\left[a_{i j}\right]$. When we do this, it is understood that $i$ is the row index and $j$ is the column index, so that $A_{i j}=a_{i j}$. If $w \in W$ we define the matrix $w[A]$ by

$$
w[A]_{i j}=w\left(A_{i j}\right) .
$$

Then $w[A B]=w[A] w[B]$ when the matrix products are defined, and $w[A]^{\top}=$ $w\left[A^{\top}\right]$. Row vectors $\mathbf{y} \in K^{l}$ are viewed as matrices $\mathbf{y}=\left[y_{1}, \ldots, y_{l}\right]$. Column vectors are viewed as matrices $\mathbf{y}^{\top}=\left[y_{1}, \ldots, y_{l}\right]^{\top}$. If $A$ is a rectangular matrix over $K$ and $\partial \in \operatorname{Der}_{K}$ we define the matrix $\partial[A]$ by

$$
\partial[A]_{i j}=\partial\left(A_{i j}\right) .
$$

Then $\partial[A B]=\partial[A] B+A \partial[B]$ when the matrix products are defined. If $\mathbf{y}=$ $\left[y_{1}, \ldots, y_{l}\right] \in K^{l}$ we let $J(\mathbf{y})$ denote the Jacobian matrix defined by

$$
J(\mathbf{y})_{i j}=\partial_{i} y_{j} .
$$

Let $R=\{f \in S \mid w f=f$ for all $w \in W\}$ be the algebra of $W$-invariant polynomial functions on $V$. As in Section 1, choose algebraically independent homogeneous polynomials $f_{1}, \ldots, f_{l} \in R$ such that $R=\mathbf{R}\left[f_{1}, \ldots, f_{l}\right]$. Let $\mathbf{f}=$ $\left[f_{1}, \ldots, f_{l}\right] \in S^{l}$. For $1 \leq j \leq l$ define $\theta_{j} \in \operatorname{Der}_{S}$ by

$$
\theta_{j}(g)=\left(d g, d f_{j}\right)
$$

for $g \in S$, where ( , ) is the bilinear form on $\Omega_{S}^{1}$ defined by (2.3). It is known [Sai1], [Ter, Theorem 2] that $D(\mathcal{A})$ is a free $S$-module with basis $\theta_{1}, \ldots, \theta_{l}$.

Let $\operatorname{Der}_{R}$ denote the $R$-module of $\mathbf{R}$-derivations of $R$ and define $\operatorname{Der}_{K}$ in similar manner. Then $\operatorname{Der}_{K}=K \partial_{1} \oplus \cdots \oplus K \partial_{l}$. Define $D^{(1)}, \ldots, D^{(l)} \in \operatorname{Der}_{R}$ by $D^{(i)} f_{j}=$ $\delta_{i j}$. Let $\operatorname{Der}_{K}$ denote the $K$-vector space of $\mathbf{R}$-derivations of $K$. We may extend $D^{(i)}: R \rightarrow R$ uniquely to an element of $\operatorname{Der}_{K}$ which we also call $D^{(i)}$. Since $D^{(i)}=\sum_{j=1}^{l}\left(D^{(i)} x_{j}\right) \partial_{j}$ we have $\delta_{i k}=D^{(i)} f_{k}=\sum_{j=1}^{l}\left(D^{(i)} x_{j}\right)\left(\partial_{j} f_{k}\right)$. Thus

$$
\left[D^{(i)} x_{j}\right]=J(\mathbf{f})^{-1}
$$


Recall, from the Introduction, that we number the exponents $m_{j}=\operatorname{deg}\left(f_{j}\right)-1$ so that $m_{1} \leq \cdots \leq m_{l}$. Since $W$ is irreducible we have $m_{l-1}<m_{l}$ [Bou, V.6.2, Corollary 2], and $m_{l}+1=h$ is the Coxeter number of $W$ [Bou, V.6.2, Theorem 1]. It follows from the inequality $m_{l-1}<m_{l}$ that the one-dimensional space $\mathbf{R} D^{(l)}$ is uniquely determined by $W$ and is independent of the choice of $f_{1}, \ldots, f_{l}$ [Sai2, (2.2)], [SYS, (1.6)]. This remark of Saito is fundamental for the proof of our theorem. We make the following

Definition 2.5. A Saito derivation is a nonzero element of $\mathbf{R} D^{(l)}$.

Thus Saito derivations are characterized by the property

$$
D f_{1}=D f_{2}=\cdots=D f_{l-1}=0, D f_{l} \in \mathbf{R}^{*} .
$$

We choose a Saito derivation $D$ and fix it throughout the paper. Define $h_{j} \in K$ for $1 \leq j \leq l$ and $\mathbf{h} \in K^{l}$ by

$$
h_{j}=D x_{j} \text { and } \mathbf{h}=\left[h_{1}, \ldots, h_{l}\right] .
$$

It follows from (2.4) that $\mathbf{h}$ is, up to constant multiple, the last row of $J(\mathbf{f})^{-1}$ :

$$
\mathbf{h} \doteq\left[J(\mathbf{f})_{l 1}^{-1}, \ldots, J(\mathbf{f})_{l l}^{-1}\right] .
$$

Here and elsewhere $\doteq$ means equality of vectors (or matrices or polynomials) up to a nonzero constant multiple. By [Bou, , Proposition 6 (ii)] we have

$$
\operatorname{det} J(\mathbf{f}) \doteq Q
$$

It follows from (2.9) that $D^{(i)}(S) \subseteq Q^{-1} S$ for $1 \leq i \leq l$. Thus

$$
h_{j} \in Q^{-1} S
$$

for $1 \leq j \leq l$. If $g \in S$ is homogeneous, we define the degree of $Q^{-1} g \in K$ by $\operatorname{deg}\left(Q^{-1} g\right)=\operatorname{deg}(g)-\operatorname{deg}(Q)=\operatorname{deg}(g)-\sum_{i=1}^{l} m_{i} ;$ the second equality follows from (2.9). From (2.8) we have

$$
\operatorname{deg}\left(h_{j}\right)=-m_{l}
$$

Define $L_{1}, \ldots, L_{l} \in \operatorname{Der}_{K}$ by

$$
L_{i}=\left[\partial_{i}, D\right]=\partial_{i} D-D \partial_{i} .
$$

Then $L_{i} x_{j}=\partial_{i} D x_{j}-D \partial_{i} x_{j}=\partial_{i} D x_{j}-D \delta_{i j}=\partial_{i} D x_{j}=\partial_{i} h_{j}$ so that

$$
J(\mathbf{h})=\left[L_{i} x_{j}\right] .
$$


Define a matrix $N \in \mathbf{M}_{l}(S)$ by

$$
N_{i j}=\left(d x_{i}, d f_{j}\right)=\theta_{j}\left(x_{i}\right) .
$$

Let

$$
\Gamma=\left[\left(x_{i}, x_{j}\right)\right]
$$

be the matrix of the form $($,$) on V^{*}$ with respect to the basis $x_{1}, \ldots, x_{l}$. Then

$$
N=\Gamma J(\mathbf{f}) .
$$

Thus, if $x_{1}, \ldots, x_{l}$ is an orthonormal basis then $N=J(\mathbf{f})$. Define a matrix $B \in$ $\mathbf{M}_{l}(K)$ by

$$
B=-N^{\top} J(\mathbf{h}) J(\mathbf{f})=-J(\mathbf{f})^{\top} \Gamma J(\mathbf{h}) J(\mathbf{f}) .
$$

The matrices $J(\mathbf{h})$ and $B$ are the key constructs in our argument. Note that $\mathbf{h}$ depends only on the chosen derivation $D$ and not on the chosen basic invariants $f_{1}, \ldots, f_{l}$. On the other hand $B$ does depend on $f_{1}, \ldots, f_{l}$. We will prove in Corollary 3.33 that if $W$ is not of type $\mathrm{D}_{l}$ with $l$ even, and we replace $D$ by $-D$ if necessary, then it is possible to choose a basis $x_{1}, \ldots, x_{l}$ for $V^{*}$ and basic invariants $f_{1}, \ldots, f_{l}$ so that $B$ has the form

$$
B=\left[\begin{array}{ccccc}
0 & 0 & \cdots & 0 & m_{l} \\
0 & 0 & \cdots & m_{l-1} & * \\
\vdots & \vdots & & \vdots & \vdots \\
0 & m_{2} & \cdots & * & * \\
m_{1} & * & \cdots & * & *
\end{array}\right] .
$$

where the entries $*$ lie in $R$. The reason for the possible sign change in $D$ will become clear in the proof of Corollary 3.33. In Section 5 we give examples of a matrix $B$ of the form (2.18) in case $l=2$ and in case $W$ has type $\mathrm{B}_{l}$.

Remark 2.19. K. Saito introduced the concept of flat generators for the ring of polynomial invariants of an irreducible real reflection group $W$ [Sai2]. A system of basic invariants $f_{1}, \ldots, f_{l}$ is called a system of flat generators if the matrix $D\left[\left(d f_{i}, d f_{j}\right)\right]$ is a constant matrix. It is known [Sai2] that the space $\mathbf{R} f_{1}+\cdots+\mathbf{R} f_{l}$ is uniquely determined by $W$. In [SYS], Saito, Yano and Sekiguchi explicitly determined a system of flat generators for each irreducible Coxeter group except $\mathrm{E}_{7}$ and $\mathrm{E}_{8}$. We will see in $(3.28)$ that $D\left[\left(d f_{i}, d f_{j}\right)\right]=B+B^{\top}$. So the matrix $B$ may be regarded as a refinement of $D\left[\left(d f_{i}, d f_{j}\right)\right]$ in the sense that $B$ determines $D\left[\left(d f_{i}, d f_{j}\right)\right]$. The study of $B$ therefore seems intriguing. For example we do not know if $B$ is a constant matrix for a system of flat generators. It is known [Sai2, (5.1)], [SYS, (1.12)] that $D\left[\left(d f_{i}, d f_{j}\right)\right]$ is an invertible matrix. The invertibility is important because it gives a linear structure on the quotient variety $V / W=$ $\operatorname{Spec} \mathbf{R}\left[f_{1}, \ldots, f_{l}\right]$ [Sai2]. In Lemma 3.9 we will show that $B$ is also invertible. 


\section{$\S$ 3. Invertibility of $B$ and $J(\mathbf{h})$}

In this Section we will prove that the entries of $B$ are $W$-invariant polynomials and that $\operatorname{det} B \in R^{*}$ is a unit. It follows then from (2.9), (2.16) and (2.17) that $\operatorname{det} J(\mathbf{h}) \doteq Q^{-2}$.

Lemma 3.1. $B \in \mathbf{M}_{l}(R)$.

Proof. Let $\rho: W \rightarrow \mathbf{G L}_{l}(\mathbf{R})$ be the matrix representation of $W$ afforded by the $W$-module $V$ relative to the basis $e_{1}, \ldots, e_{l}$. Thus

$$
w e_{j}=\sum_{i=1}^{l} \rho(w)_{i j} e_{i} \text { and } w x_{j}=\sum_{i=1}^{l} \rho\left(w^{-1}\right)_{j i} x_{i}
$$

Since $w \partial_{v}=\partial_{w v}$ for $w \in W$ and $v \in V$ we also have

$$
w \partial_{j}=\sum_{i=1}^{l} \rho(w)_{i j} \partial_{i}
$$

To prove that the entries of $B$ are $W$-invariant we need transformation rules for the action of $w \in W$ on certain matrices defined by basic invariants $f_{1}, \ldots, f_{l}$. These rules are

$$
\begin{gathered}
w[N]=\rho\left(w^{-1}\right) N, \\
w[J(\mathbf{f})]=\rho(w)^{\top} J(\mathbf{f}), \\
w[\mathbf{h}]=\mathbf{h} \rho\left(w^{-1}\right)^{\top}, \\
w[J(\mathbf{h})]=\rho(w)^{\top} J(\mathbf{h}) \rho\left(w^{-1}\right)^{\top} .
\end{gathered}
$$

We sketch the proofs of these formulas. To prove (3.4) note that $w[N]_{i j}=$ $w\left(N_{i j}\right)=w\left(\left(d x_{i}, d f_{j}\right)\right)=\left(w\left(d x_{i}\right), w\left(d f_{j}\right)\right)=\left(d\left(w x_{i}\right), d\left(w f_{j}\right)\right)=\left(d\left(w x_{i}\right), d f_{j}\right)=$ $\sum_{k=1}^{l} \rho\left(w^{-1}\right)_{i k}\left(d x_{k}, d f_{j}\right)=\left(\rho\left(w^{-1}\right) N\right)_{i j}$. To prove $(3.5)$, note that $w[\mathbf{f}]=\mathbf{f}$ and use (3.3). To prove (3.6), note that $w\left[J(\mathbf{f})^{-1}\right]=J(\mathbf{f})^{-1} \rho\left(w^{-1}\right)^{\top}$ and use (2.8). The last transformation rule (3.7) follows from (3.6) and (3.3). It follows from (3.4)-(3.7) that $w[B]=w\left[N^{\top}\right] w[J(\mathbf{h})] w[J(\mathbf{f})]=N^{\top} J(\mathbf{h}) J(\mathbf{f})=B$. Thus the entries of $B$ are $W$-invariant. To complete the proof we must show that the entries of $B$ are polynomials. It follows from (2.13) that $L_{i}=\sum_{k=1}^{l}\left(L_{i} x_{k}\right) \partial_{k}=$ $\sum_{k=1}^{l}\left(\partial_{i} h_{k}\right) \partial_{k}$. Then $(J(\mathbf{h}) J(\mathbf{f}))_{i j}=\sum_{k}\left(\partial_{i} h_{k}\right)\left(\partial_{k} f_{j}\right)=L_{i} f_{j}=\left[\partial_{i}, D\right] f_{j}=$ $\partial_{i} D f_{j}-D \partial_{i} f_{j}=-D \partial_{i} f_{j}=-D\left[J(\mathbf{f})_{i j}\right]$. This shows that

$$
J(\mathbf{h}) J(\mathbf{f})=-D[J(\mathbf{f})] .
$$

Since $J(\mathbf{f}) \in \mathbf{M}_{l}(S)$, it follows from (2.10) that $D[J(\mathbf{f})] \in \mathbf{M}_{l}\left(Q^{-1} S\right)$, so $Q B \in$ $\mathbf{M}_{l}(S)$. Since $B_{i j} \in R$ and $Q$ is an anti-invariant polynomial, it follows that $Q B_{i j}$ 
is an anti-invariant polynomial and hence [Bou, V.5.5, Proposition 6(iv)] lies in $Q R$. Thus $B_{i j} \in R$.

The next lemma asserts, in particular, that $\operatorname{det} B$ is a non-zero real number.

Lemma 3.9. 1) If $W$ is not of type $\mathrm{D}_{l}$ with $l$ even then

$$
B=\left[\begin{array}{ccccc}
0 & 0 & \ldots & 0 & B_{1 l} \\
0 & 0 & \ldots & B_{2, l-1} & B_{2 l} \\
\vdots & \vdots & & \vdots & \vdots \\
0 & B_{l-1,2} & & B_{l-1, l-1} & B_{l-1, l} \\
B_{l 1} & B_{l 2} & \ldots & B_{l, l-1} & B_{l l}
\end{array}\right]
$$

where

$$
\begin{array}{r}
B_{i j}=0 \text { if } i+j<l+1 \\
B_{i j} \in \mathbf{R}^{*} \text { if } i+j=l+1 \\
m_{i} B_{i j}=m_{j} B_{j i} \text { if } i+j=l+1 .
\end{array}
$$

2) If $W$ is of type $\mathrm{D}_{l}$ with $l=2 k$ then the $2 \times 2$ block in rows and columns $k, k+1$ of the matrix (3.10) - the center of the matrix - is to be replaced by a $2 \times 2$ block

$$
B_{0}=\left[\begin{array}{cc}
B_{k, k} & B_{k, k+1} \\
B_{k+1, k} & B_{k+1, k+1}
\end{array}\right]
$$

with constant entries, where $B_{k, k+1}=B_{k+1, k}$ and $\operatorname{det} B_{0} \in \mathbf{R}^{*}$. The statement (3.11) still holds true outside the $2 \times 2$ block $B_{0}$.

Proof. We agree in this argument that summation indices range over $1, \ldots, l$. From (2.17) and (3.8) we have

$$
B=J(\mathbf{f})^{\top} \Gamma D[J(\mathbf{f})] .
$$

If $y \in K$ let $\operatorname{grad}(y)=\left[\partial_{1} y, \ldots, \partial_{l} y\right] \in K^{l}$ denote the gradient vector and let $\operatorname{Hess}(y) \in \mathbf{M}_{l}(K)$ denote the Hessian matrix, defined by Hess $(y)_{i j}=\partial_{i} \partial_{j} y$. Then

$$
\begin{aligned}
B_{i j} & =\sum_{p, q}\left(\partial_{p} f_{i}\right)\left(d x_{p}, d x_{q}\right) D\left(\partial_{q} f_{j}\right) \\
& =\sum_{p, q}\left(\partial_{p} f_{i}\right)\left(d x_{p}, d x_{q}\right) \sum_{r} h_{r}\left(\partial_{r} \partial_{q} f_{j}\right) \\
& =\sum_{p, q, r}\left(\partial_{p} f_{i}\right)\left(d x_{p}, d x_{q}\right)\left(\partial_{q} \partial_{r} f_{j}\right) h_{r} \\
& =\operatorname{grad}\left(f_{i}\right) \Gamma \operatorname{Hess}\left(f_{j}\right) \mathbf{h}^{\top} .
\end{aligned}
$$


It follows from (3.14) and (2.11) that

$$
\operatorname{deg}\left(B_{i j}\right)=m_{i}+m_{j}-1-m_{l}=m_{i}+m_{j}-h
$$

when $B_{i j} \neq 0$. Thus $B_{i j}=0$ whenever $m_{i}+m_{j}<h$. Also $B_{i j} \in \mathbf{R}$ by (3.15) whenever $m_{i}+m_{j}=h$.

We remark, parenthetically, that if $i+j \leq l$ then $j<l-i+1$ so $m_{i}+m_{j} \leq$ $m_{i}+m_{l-i+1}=h$ by duality in the exponents [Bou, V.6.2]. If equality holds in the last formula then, since $j \neq l-i+1$, it follows from list of exponents in [Bou, VI.4] that $W$ is of type $\mathrm{D}_{l}$ with $l$ even. Thus in Case 1) the matrix $B$ has the form (3.10). We do not know, at this stage, that the entries $B_{i, l-i+1}$ on the second diagonal are nonzero. Now return to the main line of argument. Define the row vector

$$
\mathbf{g}^{(i, j)}=\operatorname{grad}\left(f_{i}\right) \Gamma \operatorname{Hess}\left(f_{j}\right) \in S^{l} .
$$

Then $\operatorname{deg}\left(\mathbf{g}^{(i, j)}\right)=m_{i}+m_{j}-1$ when $\mathbf{g}^{(i, j)} \neq 0$. By arguments like those used in the proofs of (3.4)-(3.7) we have the following transformation rules:

$$
\begin{gathered}
\Gamma=w[\Gamma]=\rho\left(w^{-1}\right) \Gamma \rho\left(w^{-1}\right)^{\top}, \\
w\left[\operatorname{grad}\left(f_{i}\right)\right]=\operatorname{grad}\left(f_{i}\right) \rho(w), \\
w\left[\operatorname{Hess}\left(f_{j}\right)\right]=\rho(w)^{\top} \operatorname{Hess}\left(f_{j}\right) \rho(w) .
\end{gathered}
$$

From these transformation rules, we have

$$
w\left[\mathbf{g}^{(i, j)}\right]=\mathbf{g}^{(i, j)} \rho(w) .
$$

If $\mathbf{g}=\left[g_{1}, \ldots, g_{l}\right] \in S^{l}$ and $w[\mathbf{g}]=\mathbf{g} \rho(w)$ for all $w \in W$ then $g_{1} d x_{1}+\cdots+g_{l} d x_{l}=$ $[\mathbf{g}]\left[d x_{1}, \ldots, d x_{l}\right]^{\top}$ is $W$-invariant. It is shown in [Sol1, Theorem] that every $W$ invariant 1-form $g_{1} d x_{1}+\cdots+g_{l} d x_{l}$ with $g_{i} \in S$ lies in $\sum_{k} R d f_{k}$. Thus, by (3.20), we may write

$$
\mathbf{g}^{(i, j)}=\sum_{k} r_{k}^{(i, j)} \operatorname{grad}\left(f_{k}\right)
$$

with homogeneous $r_{k}^{(i, j)} \in R$. It follows from (3.14) and (3.15) that

$$
B_{i j}=\mathbf{g}^{(i, j)} \mathbf{h}^{\top}=\sum_{k} r_{k}^{(i, j)} \operatorname{grad}\left(f_{k}\right) \mathbf{h}^{\top}=\sum_{k} r_{k}^{(i, j)} D f_{k}=r_{l}^{(i, j)} D f_{l}
$$

and

$$
\operatorname{deg}\left(r_{k}^{(i, j)}\right)=m_{i}+m_{j}-m_{k}-1
$$

when $r_{k}^{(i, j)} \neq 0$. Let $\mathbf{x}=\left[x_{1}, \ldots, x_{l}\right]$. Since $\operatorname{deg}\left(\partial_{j} f_{i}\right)=m_{i}$ for $1 \leq j \leq l$, it follows from (3.16) and the Euler formula that

$$
\begin{aligned}
& m_{i} \mathbf{g}^{(i, j)} \mathbf{x}^{\top}=m_{i} \operatorname{grad}\left(f_{i}\right) \Gamma m_{j} \operatorname{grad}\left(f_{j}\right)^{\top} \\
= & m_{j} \operatorname{grad}\left(f_{j}\right) \Gamma m_{i} \operatorname{grad}\left(f_{i}\right)^{\top}=m_{j} \mathbf{g}^{(j, i)} \mathbf{x}^{\top}
\end{aligned}
$$


because $\Gamma$ is a symmetric matrix. On the other hand, by (3.21) and the Euler formula, we have

$$
m_{i} \mathbf{g}^{(i, j)} \mathbf{x}^{\top}=m_{i} \sum_{k} r_{k}^{(i, j)} \operatorname{grad}\left(f_{k}\right) \mathbf{x}^{\top}=m_{i} \sum_{k} r_{k}^{(i, j)}\left(m_{k}+1\right) f_{k}
$$

Combine (3.25) with (3.24). This gives

$$
m_{i} \sum_{k} r_{k}^{(i, j)}\left(m_{k}+1\right) f_{k}=m_{j} \sum_{k} r_{k}^{(j, i)}\left(m_{k}+1\right) f_{k}
$$

for all $1 \leq i, j \leq l$. It follows from (3.23) that both sides of (3.26) are homogeneous polynomials of degree $m_{i}+m_{j}$. Suppose now that $i, j$ satisfy $m_{i}+m_{j}=h=$ $\operatorname{deg}\left(f_{l}\right)$. Then $\operatorname{deg}\left(r_{k}^{(i, j)}\right)<h$ and thus $r_{k}^{(i, j)} \in \mathbf{R}\left[f_{1}, \ldots, f_{l-1}\right]$. Since the invariant polynomials $f_{1}, \ldots, f_{l}$ are algebraically independent, we can equate the coefficients of $f_{l}$ on both sides of (3.26) and conclude that $m_{i} r_{l}^{(i, j)}\left(m_{l}+1\right)=m_{j} r_{l}^{(j, i)}\left(m_{l}+1\right)$. This proves

$$
m_{i} B_{i j}=m_{j} B_{j i} \text { whenever } m_{i}+m_{j}=h
$$

because of (3.22). Note that $B_{i j} \in \mathbf{R}$ by (3.15) whenever $m_{i}+m_{j}=h$. On the other hand

$$
B+B^{\top}=J(\mathbf{f})^{\top} \Gamma D[J(\mathbf{f})]+D\left[J(\mathbf{f})^{\top}\right] \Gamma J(\mathbf{f})=D\left[J(\mathbf{f})^{\top} \Gamma J(\mathbf{f})\right]=D\left[\left(d f_{i}, d f_{j}\right)\right]
$$

where the last equality follows from (2.3). The matrix on the right is non-singular, as shown in [Sai2, (5.1)], [SYS, (1.13)]. Thus

$$
\operatorname{det}\left(B+B^{\top}\right) \neq 0
$$

Case 1) Assume that $W$ is not of type $\mathrm{D}_{l}$ with $l$ even. If $i+j<l+1$, then $m_{i}+m_{j}<h$ and thus, as we have already remarked, $B_{i j}=0$. Note that

$$
\operatorname{det}\left(B+B^{\top}\right) \doteq \prod_{i+j=l+1}\left(B_{i j}+B_{j i}\right)
$$

By (3.29) and (3.30) we have $B_{i j}+B_{j i} \neq 0$ whenever $i+j=l+1$. It follows from (3.27) that $B_{i j} \neq 0$ whenever $i+j=l+1$. This proves the desired result in Case 1).

Case 2) Assume that $W$ is of type $\mathrm{D}_{l}$ with $l=2 k$ even. If $i+j<l+1$ with $(i, j) \neq(k, k)$, then $m_{i}+m_{j}<h$ and thus $B_{i j}=0$. Let $B_{0}$ be as in (3.12). Then

$$
\operatorname{det}\left(B+B^{\top}\right) \doteq \operatorname{det}\left(B_{0}+B_{0}^{\top}\right) \prod\left(B_{i j}+B_{j i}\right),
$$

where the product is over the set $\{(i, j) \mid i+j=l+1$ and $|i-j|>1\}$. By (3.29) we have $B_{i j}+B_{j i} \neq 0$ whenever $i+j=l+1$ and $|i-j|>1$. It follows from (3.27) 
that $B_{i j} \neq 0$ whenever $i+j=l+1$ and $|i-j|>1$. Since $m_{k}=m_{k+1}$, we have $B_{k, k+1}=B_{k+1, k}$ by (3.27). Thus $B_{0}$ is a symmetric matrix. By (3.29) and (3.31), we have $4 \operatorname{det} B_{0}=\operatorname{det}\left(2 B_{0}\right)=\operatorname{det}\left(B_{0}+B_{0}^{\top}\right) \neq 0$. Thus $\operatorname{det} B_{0} \neq 0$.

Corollary 3.32. The matrix $J(\mathbf{h})$ has determinant $\operatorname{det} J(\mathbf{h}) \doteq Q^{-2}$. Thus $J(\mathbf{h})$ is invertible and $h_{1}, \ldots, h_{l}$ are algebraically independent.

Proof. We have $\operatorname{det} J(\mathbf{h})=(\operatorname{det} \Gamma)^{-1}(\operatorname{det} J(\mathbf{f}))^{-2}(\operatorname{det} B) \doteq Q^{-2}$ by $(2.17),(2.9)$ and Lemma 3.9.

Corollary 3.33. If $W$ is not of type $\mathrm{D}_{l}$ with $l$ even, then it is possible to choose a Saito derivation $D$ and basic invariants $f_{1}, \ldots, f_{l}$ so that $B$ has the form (2.18).

Proof. Choose any basis $x_{1}, \ldots, x_{l}$, and basic invariants $f_{1}, \ldots, f_{l}$. Then $B$ has the form (3.10) where

$$
m_{i} B_{i, l+1-i}=m_{l+1-i} B_{l+1-i, i} \text { for } 1 \leq i \leq l .
$$

Suppose first that $l=2 k$ is even. Define $c_{i}=m_{i} / B_{l+1-i, i}$ for $1 \leq i \leq k$ and $c_{i}=1$ for $k+1 \leq i \leq l$. Then $c_{i} c_{l+1-i} B_{l+1-i, i}=m_{i}$ for $1 \leq i \leq l$ by (3.34). Define $f_{i}^{\prime}=c_{i} f_{i}$ for $1 \leq i \leq l$ and let $\mathbf{f}^{\prime}=\left[f_{1}^{\prime}, \ldots, f_{l}^{\prime}\right]$. Let $B^{\prime}=-\bar{J}\left(\mathbf{f}^{\prime}\right)^{\top} \Gamma J(\mathbf{h}) J\left(\mathbf{f}^{\prime}\right)$ and let $C=\operatorname{diag}\left(c_{1}, \ldots, c_{l}\right)$. Since $J\left(\mathbf{f}^{\prime}\right)=J(\mathbf{f}) C$ we have $B^{\prime}=C B C$ so $B_{l+1-i, i}^{\prime}=$ $c_{i} c_{l+1-i} B_{l+1-i, i}=m_{i}$. Thus replacement of $\mathbf{f}$ by $\mathbf{f}^{\prime}$ gives us (2.18). If $l=2 k+1$ is odd we must modify the argument slightly. Note that the condition (3.34) is vacuous for $i=k+1$. If $B_{k+1, k+1}<0$ we replace $D$ by $-D$. Thus, by (3.13), we may assume that $B_{k+1, k+1}>0$. Define $c_{i}=m_{i} / B_{l+1-i, i}$ for $1 \leq i \leq k$ and $c_{i}=1$ for $k+2 \leq i \leq l$ by analogy with the case $l=2 k$. Choose $c_{k+1}$ so that $c_{k+1}^{2} B_{k+1, k+1}=m_{k+1}$. Let $f_{i}^{\prime}=c_{i} f_{i}$ for $1 \leq i \leq l$. Then $D$ and the basic invariants $f_{1}^{\prime}, \ldots, f_{l}^{\prime}$ have the desired property.

We use the fact that $B$ is invertible to give the following alternative expression for the matrix $P$ in Theorem 1.4.

Proposition 3.35. Define $A=B^{-1} \in M_{l}(R)$. Then

$$
P=-N A N^{\top}
$$

Proof. By (2.16) and (2.17) we have $N=\Gamma J(\mathbf{f})$ and $B=-N^{\top} J(\mathbf{h}) J(\mathbf{f})$. Thus $N A N^{\top}=N B^{-1} N^{\top}=-\Gamma J(\mathbf{f}) J(\mathbf{f})^{-1} J(\mathbf{h})^{-1}\left(N^{\top}\right)^{-1} N^{\top}=-\Gamma J(\mathbf{h})^{-1}=-P$. 


\section{$\S$ 4. Proof of Theorem 1.4}

In this Section we will prove Theorem 1.4. We will also determine the graded $W$-module structure of $E(\mathcal{A})$. It turns out that its homogeneous component of degree $h$ is $W$-isomorphic to $V^{*}$.

Recall that the matrix $P=\Gamma J\left(h_{1}, \ldots, h_{l}\right)^{-1}$ of Theorem 1.4 is defined using a basis $e_{1}, \ldots, e_{l}$ for $V$ and the dual basis $x_{1}, \ldots, x_{l}$ for $V^{*}$ together with Saito's derivation $D$. We will study how $P$ is transformed if $e_{1}, \ldots, e_{l}$ is replaced by another basis for $V$. Suppose a basis $e_{1}^{\prime}, \ldots, e_{l}^{\prime}$ for $V$ is connected with $e_{1}, \ldots, e_{l}$ through an invertible matrix $M \in \mathbf{G L}_{l}(\mathbf{R})$ :

$$
e_{j}^{\prime}=\sum_{i=1}^{l} M_{i j} e_{i} .
$$

The new objects, which are defined using the new basis $e_{1}^{\prime}, \ldots, e_{l}^{\prime}$, will be denoted by $x_{j}^{\prime}, \partial_{j}^{\prime}, \Gamma^{\prime}$ etc.. As in (3.2)-(3.7) and (3.17)-(3.19), we have

$$
\begin{gathered}
x_{j}^{\prime}=\sum_{i=1}^{l}\left(M^{-1}\right)_{j i} x_{i}, \partial_{j}^{\prime}=\sum_{i=1}^{l} M_{i j} \partial_{i}, \\
\Gamma^{\prime}=M^{-1} \Gamma\left(M^{\top}\right)^{-1}, \mathbf{h}^{\prime}=\mathbf{h}\left(M^{\top}\right)^{-1}, J^{\prime}\left(\mathbf{h}^{\prime}\right)=M^{\top} J(\mathbf{h})\left(M^{\top}\right)^{-1} .
\end{gathered}
$$

Thus

$$
P^{\prime}=M^{-1} P\left(M^{\top}\right)^{-1}
$$

Recall that the derivations $\xi_{1}, \ldots, \xi_{l} \in \operatorname{Der}_{S}$ of Theorem 1.4 are defined by $\xi_{j}=$ $\sum_{i=1}^{l} p_{i j} \partial_{i}$ where $p_{i j}$ is the $(i, j)$ entry of $P$. By (4.2) and (4.4), we have

$$
\xi_{j}^{\prime}=\sum_{i=1}^{l}\left(M^{-1}\right)_{j i} \xi_{i} .
$$

In other words, $\xi_{1}, \ldots, \xi_{l}$ satisfy the same base change rule as $x_{1}, \ldots, x_{l}$.

Lemma 4.6. If $H \in \mathcal{A}$ then $\xi_{i}\left(\alpha_{H}\right) \in S \alpha_{H}^{2}$. Thus $\xi_{i} \in E(\mathcal{A})$ for $1 \leq i \leq l$.

Proof. Because of (4.5), we may assume that $\alpha_{H}=x_{1}$ and that $x_{1}, \ldots, x_{l}$ is an orthonormal basis. Then $P=J(\mathbf{h})^{-1}$. It is thus enough to show that each entry of the first row of $P$ is divisible by $x_{1}^{2}$. Since $x_{1}, \ldots, x_{l}$ is an orthonormal basis we have $\theta_{j}=\sum_{i=1}^{l}\left(\partial_{i} f_{j}\right) \partial_{i} \in D(\mathcal{A})$, as remarked in the Introduction. Thus $\partial_{1} f_{j}=\theta_{j}\left(x_{1}\right) \in S x_{1}$, so each entry of the first row of $J(\mathbf{f})$ is divisible by $x_{1}$. Thus, outside the first column, each entry of $\operatorname{adj} J(\mathbf{f})$, is divisible by $x_{1}$. Since $\operatorname{det} J(\mathbf{f}) \doteq Q$ is divisible by $x_{1}$ exactly once, each entry of $J(\mathbf{f})^{-1} \doteq Q^{-1}$ adj $J(\mathbf{f})$, outside the first column, has no pole along $x_{1}=0$. In particular, $h_{j}(2 \leq j \leq l)$ has 
no pole along $x_{1}=0$. It follows that each entry of $J(\mathbf{h})$ outside the first column has no pole along $x_{1}=0$. Therefore, each entry of the first row of adj $J(\mathbf{h})$ has no pole along $x_{1}=0$. Recall that $J(\mathbf{h}) \doteq Q^{-2}$ from Corollary 3.32. This implies that each entry of the first row of $J(\mathbf{h})^{-1} \doteq Q^{2}$ adj $J(\mathbf{h})$ is divisible by $x_{1}^{2}$.

Now we may complete the proof of Theorem 1.4. By Corollary 3.32 we have

$$
\operatorname{det}\left[\xi_{j}\left(x_{i}\right)\right]=\operatorname{det} P=\operatorname{det}\left(\Gamma J(\mathbf{h})^{-1}\right) \doteq Q^{2} .
$$

By Ziegler's generalization [Zie, p.351] of Saito's criterion [Sai3, p.270], [OrT, Theorem 4.19] to multiarrangements, we can conclude that $\xi_{1}, \ldots, \xi_{l} \in E(\mathcal{A})$ form a basis for the $S$-module $E(\mathcal{A})$. This completes the proof of Theorem 1.4.

The space $E(\mathcal{A})$ inherits a grading from $\operatorname{Der}_{S}$. Let $E(\mathcal{A})_{q} \subset E(\mathcal{A})$ denote the space of homogeneous elements of degree $q$. Then $E(\mathcal{A})=\bigoplus_{q \geq 0} E(\mathcal{A})_{q}$. It follows from Theorem 1.4 that

$$
E(\mathcal{A})=S \otimes_{\mathbf{R}} E(\mathcal{A})_{h}
$$

and that

$$
E(\mathcal{A})_{h}=\bigoplus_{k} \mathbf{R} \xi_{k}
$$

Thus the $W$-module structure of $E(\mathcal{A})$ is determined by that of $E(\mathcal{A})_{h}$. The $W$ module structure of $E(\mathcal{A})_{h}$ is given by the following:

Proposition 4.7. The $\mathbf{R}$-linear map $\Xi: V^{*} \longrightarrow E(\mathcal{A})_{h}$ defined by $\Xi\left(x_{i}\right)=\xi_{i}$ for $1 \leq i \leq l$, is a $W$-isomorphism.

Proof. We have already remarked in (4.5) that $\xi_{1}, \ldots, \xi_{l}$ satisfy the same base change rule as $x_{1}, \ldots, x_{l}$. Thus the assertion follows from (4.5) with $M=\rho(w)$.

Since $W$ is assumed irreducible, it follows from Schur's lemma that an arbitrary $W$-isomorphism from $V^{*}$ to $E(\mathcal{A})_{h}$ is a nonzero constant multiple of the map $\Xi$.

Proposition 4.8. If $H \in \mathcal{A}$ then $\Xi\left(\alpha_{H}\right) \in \alpha_{H} \operatorname{Der}_{S}$.

Proof. Write $\alpha_{H}=c_{1} x_{1}+\cdots+c_{l} x_{l}$ with $c_{i} \in \mathbf{R}$. Then $\Xi\left(\alpha_{H}\right)=\sum_{k} c_{k} \Xi\left(x_{k}\right)=$ $\sum_{k} c_{k} \xi_{k}$. For $1 \leq i \leq l$, let $\mathbf{e}_{i} \in \mathbf{R}^{l}$ be the $i$-th elementary unit vector. Then, by Proposition 3.35, $\Xi\left(\alpha_{H}\right)\left(x_{i}\right)=\sum_{k} c_{k} \xi_{k}\left(x_{i}\right)=\mathbf{e}_{i} P \operatorname{grad}\left(\alpha_{H}\right)^{\top}=-\mathbf{e}_{i} N A N^{\top}$ $\operatorname{grad}\left(\alpha_{H}\right)^{\top}=-\mathbf{e}_{i} N A\left(\operatorname{grad}\left(\alpha_{H}\right) N\right)^{\top} \in S \alpha_{H}$ because $\operatorname{grad}\left(\alpha_{H}\right) N=\left[\theta_{1}\left(\alpha_{H}\right), \ldots\right.$, $\left.\theta_{l}\left(\alpha_{H}\right)\right] \in\left(S \alpha_{H}\right)^{l}$. 


\section{$\S 5$. Examples}

In this Section we will study two examples: the two-dimensional double Coxeter arrangements and the double Coxeter arrangements of type $\mathrm{B}_{l}$.

1. The two-dimensional case: Let $V$ be two dimensional Euclidean space. Let $W \subset \mathbf{G L}(V)$ be a finite irreducible reflection group. Thus $W$ is a dihedral group of order $2 n$ where $n>2$. Let $\mathcal{A}$ be the corresponding Coxeter arrangement. Choose $Q$ as in (1.1). Then $\operatorname{deg}(Q)=n$. Choose an orthonormal basis $e_{1}, e_{2}$ for $V$. Let $x_{1}, x_{2}$ be the dual basis for $V^{*}$. Then $\Gamma$ is the identity matrix. The exponents of $W$ are $m_{1}=1, m_{2}=n-1$. To construct the matrix $P$ we must find a Saito derivation. Define $\lambda \in \Omega_{S}^{1}$ by $\lambda=x_{1} d x_{1}+x_{2} d x_{2}$. Define $\sigma \in \Omega_{S}^{2}$ by $\sigma=d x_{1} \wedge d x_{2}$. Let $w \in W$. Then $w \lambda=\lambda$ and $w \sigma=\operatorname{det}(w) \sigma$. Define the star operator $*: V^{*} \rightarrow V^{*}$ by $x \wedge y=(* x, y) \sigma$ for $x, y \in V^{*}$. Extend $*$ to an $S$-module map $*: \Omega_{S}^{1} \rightarrow \Omega_{S}^{1}$ by $S$-linearity. Then $*(d f)=-\left(\partial_{2} f\right) d x_{1}+\left(\partial_{1} f\right) d x_{2}$ for $f \in S$. Since $w \sigma=\operatorname{det}(w) \sigma$ we have $w(* \theta)=\operatorname{det}(w) *(w \theta)$ for $\theta \in \Omega_{S}^{1}$. Suppose $f \in R$. Then $w(\lambda, * d f)=$ $(w \lambda, w(* d f))=\operatorname{det}(w)(\lambda, * d f)$ so $-x_{2}\left(\partial_{1} f\right)+x_{1}\left(\partial_{2} f\right)=(\lambda, * d f) \in Q R$. Define $D \in \operatorname{Der}_{K}$ by

$$
D=\frac{1}{n Q}\left(-x_{2} \partial_{1}+x_{1} \partial_{2}\right) \text {. }
$$

Then $D$ maps $R \rightarrow R$. Now let $f_{1}, f_{2}$ be basic invariants with $\operatorname{deg}\left(f_{1}\right)=2$ and $\operatorname{deg}\left(f_{2}\right)=n$. Since $\operatorname{deg}(Q)=n>2=\operatorname{deg}\left(f_{1}\right)$ we have $D f_{1}=0$. Since $\operatorname{deg}\left(f_{2}\right)=$ $n=\operatorname{deg}(Q)$ we have $D f_{2} \in \mathbf{R}$. If $D f_{2}=0$ then $D x_{1}=0=D x_{2}$, a contradiction because $D f_{k}=\left(D x_{1}\right)\left(\partial_{1} f_{k}\right)+\left(D x_{2}\right)\left(\partial_{2} f_{k}\right)$ for $k=1,2$ and $J\left(f_{1}, f_{2}\right) \neq 0$. Thus $D f_{1}=0$ and $D f_{2} \in \mathbf{R}^{*}$ so $D$ is a Saito derivation by (2.6). We use this $D$ and follow the procedure in Sections 2 and 3 to construct the matrix $P$, the derivations $\xi_{1}, \xi_{2}$ and the matrix $B$. From (2.7) we have

$$
\mathbf{h}=\frac{1}{n Q}\left[-x_{2}, x_{1}\right]
$$

We compute

$$
J(\mathbf{h})=\frac{1}{n Q^{2}}\left[\begin{array}{cc}
x_{2} Q_{1} & Q-x_{1} Q_{1} \\
-Q+x_{2} Q_{2} & -x_{1} Q_{2}
\end{array}\right]
$$

where $Q_{i}=\partial_{i} Q$ for $i=1,2$. Since $\operatorname{det} J(\mathbf{h})=(1-n) / n^{2} Q^{2}$ we have

$$
P=J(\mathbf{h})^{-1}=\frac{n}{n-1}\left[\begin{array}{cc}
x_{1} Q_{2} & Q-x_{1} Q_{1} \\
-Q+x_{2} Q_{2} & -x_{2} Q_{1}
\end{array}\right]
$$

and

$$
\begin{aligned}
\xi_{1} & =\frac{n}{n-1}\left\{x_{1} Q_{2} \partial_{1}+\left(x_{2} Q_{2}-Q\right) \partial_{2}\right\} \\
\xi_{2} & =\frac{n}{n-1}\left\{\left(Q-x_{1} Q_{1}\right) \partial_{1}-x_{2} Q_{1} \partial_{2}\right\} .
\end{aligned}
$$


By Theorem 1.4, the derivations $\xi_{1}$ and $\xi_{2}$ form a basis for $E(\mathcal{A})$. Note in $(5.2)$ that the matrix $P$ depends only upon choice of $x_{1}, x_{2}$ and $Q$. The reader may have noticed that the group $W$ is peripheral to the computations in this section. In fact we can use the derivations defined by (5.3) to prove a proposition about any central arrangement in a real two dimensional vector space $V$.

Proposition 5.4. Suppose $V$ is a real vector space of dimension 2. Let $\mathcal{A}$ be an arbitrary central arrangement in $V$ and let $Q$ be its defining polynomial. Let $n=|\mathcal{A}|=\operatorname{deg} Q$. Define an $S$-module $E(\mathcal{A})$ as in (1.3). Then the derivations $\xi_{1}$ and $\xi_{2}$ given by (5.3) form a basis for $E(\mathcal{A})$.

Proof. Note that $\operatorname{det}\left[\xi_{j}\left(x_{i}\right)\right]=\operatorname{det} P \doteq Q^{2}$. Thanks to Ziegler's generalization [Zie, p.351] of Saito's criterion [Sai3, p.270], [OrT, Theorem 4.19] to multiarrangements, it is enough to show that $\xi_{i} \in E(\mathcal{A})$ for $i=1,2$. Let $H \in \mathcal{A}$. Write $\alpha_{H}=a x_{1}+b x_{2}$ with $a, b \in \mathbf{R}$. Then

$$
\xi_{1}\left(\alpha_{H}\right) \doteq a x_{1} Q_{2}+b\left(x_{2} Q_{2}-Q\right)=\alpha_{H} Q_{2}-b Q=\alpha_{H}^{2} \partial_{2}\left(Q / \alpha_{H}\right) \in \alpha_{H}^{2} S .
$$

So $\xi_{1} \in E(\mathcal{A})$. Similarly $\xi_{2} \in E(\mathcal{A})$.

Now we return to the case of Coxeter arrangements. Since $f_{1} \doteq x_{1}^{2}+x_{2}^{2}$ we may choose $f_{1}=\left(x_{1}^{2}+x_{2}^{2}\right) / 2$. If $n$ is even, then the invariant $f_{2}$ is not uniquely determined up to a constant multiple. We make a special choice of $f_{2}$. Define

$$
f_{2}=-Q(D Q) \text {. }
$$

We will find the matrix $B=J(\mathbf{f})^{\top} \Gamma D[J(\mathbf{f})]$ in (3.13) and check that $f_{1}, f_{2}$ is a system of flat generators in the sense of K. Saito; see Remark 2.19. First note that $f_{2}$ is an invariant because $Q^{2} \in R$ and $D: R \rightarrow R$ since $D$ is a Saito derivation. Since the Laplacian $\Delta=\partial_{1}^{2}+\partial_{2}^{2}$ commutes with the action of $W$, and $Q$ is an anti-invariant, $\Delta Q$ is also an anti-invariant. Since $Q$ is an anti-invariant of minimal degree, we have

$$
0=\Delta Q=Q_{11}+Q_{22} \text {. }
$$

To compute $J(\mathbf{f})$ use (5.5). Calculate $n \partial_{1} f_{2}=-\partial_{1}\left(-x_{2} Q_{1}+x_{1} Q_{2}\right)=x_{2} Q_{11}-$ $Q_{2}-x_{1} Q_{12}=-\left(x_{2} Q_{22}+Q_{2}+x_{1} Q_{12}\right)=-\left((n-1) Q_{2}+Q_{2}\right)=-n Q_{2}$. Thus $\partial_{1} f_{2}=-Q_{2}$. Similarly $\partial_{2} f_{2}=Q_{1}$. Thus

$$
J(\mathbf{f})=\left[\begin{array}{cc}
x_{1} & -Q_{2} \\
x_{2} & Q_{1}
\end{array}\right] .
$$

To compute $D[J(\mathbf{f})]$ use (5.1) and (5.5). Calculate $n Q\left(D Q_{1}\right)=-x_{2} Q_{11}+x_{1} Q_{12}=$ $x_{2} Q_{22}+x_{1} Q_{12}=(n-1) Q_{2}$. Thus $D Q_{1}=(n-1) Q_{2} / n Q$. Similarly $D Q_{2}=$ $-(n-1) Q_{1} / n Q$. Thus

$$
B=J(\mathbf{f})^{\top} D[J(\mathbf{f})]=\left[\begin{array}{cc}
x_{1} & x_{2} \\
-Q_{2} & Q_{1}
\end{array}\right] \frac{1}{n Q}\left[\begin{array}{cc}
-x_{2} & (n-1) Q_{1} \\
x_{1} & (n-1) Q_{2}
\end{array}\right]=\left[\begin{array}{cc}
0 & n-1 \\
1 & 0
\end{array}\right],
$$


and

$$
A=B^{-1}=\left[\begin{array}{cc}
0 & 1 \\
\frac{1}{n-1} & 0
\end{array}\right]
$$

The alternative expression of $P$ given in Proposition 3.35 is:

$$
P=-\left[\begin{array}{cc}
x_{1} & -Q_{2} \\
x_{2} & Q_{1}
\end{array}\right]\left[\begin{array}{cc}
0 & 1 \\
\frac{1}{n-1} & 0
\end{array}\right]\left[\begin{array}{cc}
x_{1} & x_{2} \\
-Q_{2} & Q_{1}
\end{array}\right]
$$

This agrees with (5.2) via the Euler formula. By (3.28),

$$
D\left[\left(d f_{i}, d f_{j}\right)\right]=B+B^{\top}=\left[\begin{array}{ll}
0 & n \\
n & 0
\end{array}\right] .
$$

It follows that $f_{1}, f_{2}$ is a system of flat generators. Note, by $(5.5)$, that $\Delta f_{2}=$ $\partial_{1}\left(-Q_{2}\right)+\partial_{2} Q_{1}=0$. Thus $f_{2}$ is harmonic.

2. The case $\mathrm{B}_{l}$ : Let $W$ be the Coxeter group of type $\mathrm{B}_{l}$ acting on an $l$-dimensional Euclidean space $V$ by signed permutations of an orthonormal basis $e_{1}, \ldots, e_{l}$. Let $\mathcal{A}$ be the corresponding Coxeter arrangement. Let $x_{1}, \ldots, x_{l}$ be the dual basis for $V^{*}$. Then $\Gamma$ is the identity matrix. Define

$$
p_{i}=p_{i}\left(x_{1}, \ldots, x_{l}\right)=\frac{1}{i} \sum_{k=1}^{l} x_{k}^{i}
$$

for $i \geq 1$. Define $p_{0}=1$. Let $f_{i}=p_{2 i}$. We will use the basic invariants $f_{1}, \ldots, f_{l}$ to find the matrices $B, A$, and $P$. To simplify formulas we use the following notation [Mac, pps.26-27]: if $\alpha=\left(\alpha_{1}, \ldots, \alpha_{l}\right) \in \mathbf{N}^{l}$, let $A_{\alpha}=\left[x_{j}^{\alpha_{i}}\right]$ and let $a_{\alpha}=\operatorname{det} A_{\alpha}$. Then

$$
J(\mathbf{f})=\left[\begin{array}{cccc}
x_{1} & x_{1}^{3} & \cdots & x_{1}^{2 l-1} \\
\vdots & \vdots & & \vdots \\
x_{l} & x_{l}^{3} & \cdots & x_{l}^{2 l-1}
\end{array}\right]=A_{(1,3, \ldots, 2 l-1)} .
$$

Define a derivation $D \in \operatorname{Der}_{K}$ by

$$
D y=\frac{1}{a_{(1,3, \ldots, 2 l-1)}}\left|\begin{array}{ccccc}
x_{1} & x_{1}^{3} & \ldots & x_{1}^{2 l-3} & \partial_{1} y \\
\vdots & \vdots & & \vdots & \vdots \\
x_{l} & x_{l}^{3} & \ldots & x_{l}^{2 l-3} & \partial_{l} y
\end{array}\right|,
$$

for $y \in K$. Since $D f_{1}=D f_{2}=\cdots=D f_{l-1}=0$ and $D f_{l}=1, D$ is a Saito derivation. For $i \geq 0$ let

$$
c_{i}\left(x_{1}, \ldots, x_{l}\right)=\sum_{i_{1}+\cdots+i_{l}=i} x_{1}^{i_{1}} \cdots x_{l}^{i_{l}}
$$


be the $i$-th complete symmetric polynomial; it is not possible to use the now standard notation $h_{i}$ of [Mac] since $h_{i}$ has already been used. Let

$$
\tilde{c}_{i}=\tilde{c}_{i}\left(x_{1}, \ldots, x_{l}\right)=c_{i}\left(x_{1}^{2}, \ldots, x_{l}^{2}\right) .
$$

Then $\tilde{c}_{i}$ is a $W$-invariant polynomial of degree $2 i$. Define $\tilde{c}_{i}\left(x_{1}, \ldots, x_{l}\right)=0$ if $i<0$.

Lemma 5.7. The derivation $D$ satisfies $D\left(p_{2 l+2 i}\right)=\tilde{c}_{i}\left(x_{1}, \ldots, x_{l}\right)$ for $i \geq-l$.

Proof. From (5.6) we have

$$
D\left(p_{2 l+2 i}\right)=\frac{a_{(1,3, \ldots, 2 l-3,2 l+2 i-1)}}{a_{(1,3, \ldots, 2 l-1)}}=\frac{a_{(0,2, \ldots, 2 l-4,2 l+2 i-2)}}{a_{(0,2, \ldots, 2 l-2)}} .
$$

Define $\delta=(l-1, l-2, \ldots, 1,0) \in \mathbf{N}^{l}$ and define $\lambda=(i, 0, \ldots, 0,0) \in \mathbf{N}^{l}$. Then $\lambda+\delta=(l+i-1, l-2, \ldots, 1,0)$. The right hand side of $(5.8)$ is thus $a_{\lambda+\delta} / a_{\delta}$ with $x_{i}$ replaced by $x_{i}^{2}$. By [Mac, (I.3.1), (I.3.9)] we have $a_{\lambda+\delta} / a_{\delta}=c_{i}\left(x_{1}, \ldots, x_{l}\right)$. Thus the right hand side of $(5.8)$ is $\tilde{c}_{i}\left(x_{1}, \ldots, x_{l}\right)$.

Since $J(\mathbf{f})_{i j}=x_{i}^{2 j-1}$, the entries of $B=J(\mathbf{f})^{\top} D[J(\mathbf{f})]$ in (3.13) are

$$
\begin{aligned}
B_{i j} & =\sum_{k} x_{k}^{2 i-1} D\left(x_{k}^{2 j-1}\right)=(2 j-1) \sum_{k} x_{k}^{2 i+2 j-3} D\left(x_{k}\right) \\
& =(2 j-1) D\left(p_{2 i+2 j-2}\right)=(2 j-1) \tilde{c}_{i+j-l-1}\left(x_{1}, \ldots, x_{l}\right)
\end{aligned}
$$

by Lemma 5.7. Thus

$$
\begin{aligned}
B & =\left[\begin{array}{cccccc}
0 & 0 & 0 & \cdots & 0 & 2 l-1 \\
0 & 0 & 0 & \cdots & 2 l-3 & (2 l-1) \tilde{c}_{1} \\
0 & 0 & 0 & \cdots & (2 l-3) \tilde{c}_{1} & (2 l-1) \tilde{c}_{2} \\
\vdots & \vdots & \vdots & & \vdots & \vdots \\
0 & 0 & 5 & \cdots & (2 l-3) \tilde{c}_{l-4} & (2 l-1) \tilde{c}_{l-3} \\
0 & 3 & 5 \tilde{c}_{1} & \cdots & (2 l-3) \tilde{c}_{l-3} & (2 l-1) \tilde{c}_{l-2} \\
1 & 3 \tilde{c}_{1} & 5 \tilde{c}_{2} & \cdots & (2 l-3) \tilde{c}_{l-2} & (2 l-1) \tilde{c}_{l-1}
\end{array}\right] \\
& =\left[\begin{array}{cccccc}
1 & 0 & \cdots & 0 & 0 & 0 \\
\tilde{c}_{1} & 1 & \cdots & 0 & 0 & 0 \\
\tilde{c}_{2} & \tilde{c}_{1} & \cdots & 0 & 0 & 0 \\
\vdots & \vdots & & \vdots & \vdots & \vdots \\
\tilde{c}_{l-3} & \tilde{c}_{l-4} & \cdots & 1 & 0 & 0 \\
\tilde{c}_{l-2} & \tilde{c}_{l-3} & \cdots & \tilde{c}_{1} & 1 & 0 \\
\tilde{c}_{l-1} & \tilde{c}_{l-2} & \cdots & \tilde{c}_{2} & \tilde{c}_{1} & 1
\end{array}\right]\left[\begin{array}{ccccc}
0 & 0 & \cdots & 0 & 2 l-1 \\
0 & 0 & \cdots & 2 l-3 & 0 \\
\vdots & \vdots & & \vdots & \vdots \\
0 & 3 & \cdots & 0 & 0 \\
1 & 0 & \cdots & 0 & 0
\end{array}\right]
\end{aligned}
$$


On the other hand, it is known [Mac, p.21] that

$$
\begin{gathered}
{\left[\begin{array}{cccccc}
1 & 0 & \cdots & 0 & 0 & 0 \\
\tilde{c}_{1} & 1 & \cdots & 0 & 0 & 0 \\
\tilde{c}_{2} & \tilde{c}_{1} & \cdots & 0 & 0 & 0 \\
\vdots & \vdots & & \vdots & \vdots & \vdots \\
\tilde{c}_{l-3} & \tilde{c}_{l-4} & \cdots & 1 & 0 & 0 \\
\tilde{c}_{l-2} & \tilde{c}_{l-3} & \cdots & \tilde{c}_{1} & 1 & 0 \\
\tilde{c}_{l-1} & \tilde{c}_{l-2} & \cdots & \tilde{c}_{2} & \tilde{c}_{1} & 1
\end{array}\right]} \\
=\left[\begin{array}{ccccccc}
1 & \\
-\tilde{e}_{1} & & & \cdots & 0 & 0 & 0 \\
\tilde{e}_{2} & -\tilde{e}_{1} & \cdots & 0 & 0 & 0 \\
\vdots & \vdots & & & \vdots & \vdots & \vdots \\
(-1)^{l-3} \tilde{e}_{l-3} & (-1)^{l-4} \tilde{e}_{l-4} & \cdots & 1 & 0 & 0 \\
(-1)^{l-2} \tilde{e}_{l-2} & (-1)^{l-3} \tilde{e}_{l-3} & \cdots & -\tilde{e}_{1} & 1 & 0 \\
(-1)^{l-1} \tilde{e}_{l-1} & (-1)^{l-2} \tilde{e}_{l-2} & \cdots & \tilde{e}_{2} & -\tilde{e}_{1} & 1
\end{array}\right],
\end{gathered}
$$

where $\tilde{e}_{i}=\tilde{e}_{i}\left(x_{1}, \ldots, x_{l}\right)=e_{i}\left(x_{1}^{2}, \ldots, x_{l}^{2}\right)$ is the $i$-th elementary symmetric polynomial in $x_{1}^{2}, \ldots, x_{l}^{2}$. Therefore $A=B^{-1}$ is equal to

$$
\begin{gathered}
{\left[\begin{array}{ccccc}
0 & 0 & \cdots & 0 & 1 / 1 \\
0 & 0 & \cdots & 1 / 3 & 0 \\
\vdots & \vdots & & \vdots & \vdots \\
0 & 1 /(2 l-3) & \cdots & 0 & 0 \\
1 /(2 l-1) & 0 & \cdots & 0 & 0
\end{array}\right]} \\
{\left[\begin{array}{cccccc}
1 & 0 & \cdots & 0 & 0 & 0 \\
-\tilde{e}_{1} & 1 & \cdots & 0 & 0 & 0 \\
\tilde{e}_{2} & -\tilde{e}_{1} & \cdots & 0 & 0 & 0 \\
\vdots & \vdots & & \vdots & \vdots & \vdots \\
(-1)^{l-3} \tilde{e}_{l-3} & (-1)^{l-4} \tilde{e}_{l-4} & \cdots & 1 & 0 & 0 \\
(-1)^{l-2} \tilde{e}_{l-2} & (-1)^{l-3} \tilde{e}_{l-3} & \cdots & -\tilde{e}_{1} & 1 & 0 \\
(-1)^{l-1} \tilde{e}_{l-1} & (-1)^{l-2} \tilde{e}_{l-2} & \cdots & \tilde{e}_{2} & -\tilde{e}_{1} & 1
\end{array}\right] .}
\end{gathered}
$$

Since $\Gamma$ is the identity matrix we have $N=J(\mathbf{f})$. Thus, by Proposition 3.35, the matrix $P$ in Theorem 1.4 is given by

$$
P=-N A N^{\top}=-J(\mathbf{f}) A J(\mathbf{f})^{\top}
$$

with $A$ as above. 


\section{$\S$ 6. Anti-invariant differential 1-forms}

If $M$ is an $\mathbf{R}[W]$-module let $M^{W}=\{x \in M \mid w x=x$ for all $w \in W\}$ denote the space of invariant elements in $M$. Let $M^{\operatorname{det}}=\{x \in M \mid w x=\operatorname{det}(w) x$ for all $w \in$ $W\}$ denote the space of anti-invariant elements in $M$. In this section we use the fact that $\operatorname{det} J(\mathbf{h}) \neq 0$ to prove the following Proposition. Recall that $D$ denotes a Saito derivation and that $h_{i}=D x_{i}$.

Proposition 6.1. Let $W \subset \mathbf{G L}(V)$ be a finite irreducible group generated by reflections. Let $u_{i}=Q h_{i}$. Define an $\mathbf{R}$-linear map $\hat{d}: S \rightarrow \Omega_{S}^{1}$ by

$$
\hat{d} f=\sum_{i=1}^{l}\left(\partial_{i} f\right) d u_{i}
$$

for $f \in S^{l}$. Let $f_{1}, \ldots, f_{l}$ be basic invariants. Then

$$
\left(\Omega_{S}^{1}\right)^{\operatorname{det}}=R \hat{d} f_{1} \oplus \cdots \oplus R \hat{d} f_{l} .
$$

Proof. Choose an orthonormal basis $x_{1}, \ldots, x_{l}$ for $V^{*}$. By Corollary $3.32, h_{1}, \ldots, h_{l}$ are algebraically independent. By $(2.10)$ we have $Q h_{i} \in S$. Let $\mathbf{u}=\left[u_{1}, \ldots, u_{l}\right] \in$ $S^{l}$. Since $\mathbf{u}=Q \mathbf{h}$ it follows that $u_{1}, \ldots, u_{l}$ are algebraically independent. Thus $\operatorname{det} J(\mathbf{u}) \neq 0$. To show that $\hat{d} f_{i} \in\left(\Omega_{S}^{1}\right)^{\text {det }}$ we must check

$$
w(\hat{d} f)=\operatorname{det}(w) \hat{d}(w f)
$$

for all $w \in W$ and $f \in S$. Let $\mathbf{x}=\left[x_{1}, \ldots, x_{l}\right]$. Let $\rho: W \rightarrow \mathbf{G L}_{l}(\mathbf{R})$ be the matrix representation of $W$ defined in (3.2). If $w \in W$ then $w[\mathbf{x}]=\mathbf{x} \rho\left(w^{-1}\right)^{\top}$. Since $Q$ is anti-invariant and $\mathbf{u}=Q \mathbf{h}$, it follows from (3.6) that $w[\mathbf{u}]=\operatorname{det}(w) \mathbf{u} \rho\left(w^{-1}\right)^{\top}$. Thus $w\left(\hat{d} x_{j}\right)=\operatorname{det}(w) \hat{d}\left(w x_{j}\right)$ for $j=1, \ldots, l$. This proves (6.3) for $f=x_{j}$. Since the map $f \mapsto \hat{d} f$ is R-linear and $\hat{d}(f g)=f \hat{d} g+g \hat{d} f$, for all $f, g \in S$, the set of all $f \in S$ which satisfy (6.3) is an $\mathbf{R}$-subalgebra of $S$ which contains $x_{1}, \ldots, x_{l}$ and is thus equal to $S$. This proves (6.3). Thus $\left(\Omega_{S}^{1}\right)^{\operatorname{det}} \supseteq R \hat{d} f_{1}+\cdots+R \hat{d} f_{l}$.

Now argue as in [Sol2, Theorem 3] to show that $\hat{d} f_{1}, \ldots, \hat{d} f_{l}$ are linearly independent over $S$. If not, then we have a relation $\sum_{i=1}^{l} g_{i} \hat{d} f_{i}=0$ where $g_{i} \in S$ and $g_{1}$, say, is not zero. Multiply the relation by $\hat{d} f_{1}$. This gives $\hat{d} f_{1} \wedge \cdots \wedge \hat{d} f_{l}=0$. Let $\mathbf{f}=\left[f_{1}, \ldots, f_{l}\right]$. It follows from (6.2) that

$$
\hat{d} f_{1} \wedge \cdots \wedge \hat{d} f_{l}=\operatorname{det}(J(\mathbf{f})) \operatorname{det}(J(\mathbf{u})) d x_{1} \wedge \cdots \wedge d x_{l},
$$

which is not zero since $\operatorname{det} J(\mathbf{u}) \neq 0$. This contradiction proves the linear independence. Thus the sum $R \hat{d} f_{1}+\cdots+R \hat{d} f_{l}$ is direct, so

$$
\left(\Omega_{S}^{1}\right)^{\operatorname{det}} \supseteq R \hat{d} f_{1} \oplus \cdots \oplus R \hat{d} f_{l} .
$$


To prove equality in (6.4) we show that both graded vector spaces have the same Poincaré series. Let $n=\operatorname{deg}(Q)$. By $(2.11)$ we have $\operatorname{deg}\left(u_{i}\right)=n-m_{l}$ for $1 \leq i \leq l$. Thus $\operatorname{deg}\left(d u_{i}\right)=n-h$ where $h$ is the Coxeter number. Since $\operatorname{deg}\left(d f_{i}\right)=m_{i}$ we have

$$
\operatorname{Poin}\left(\bigoplus_{i=1}^{l} R \hat{d} f_{i}, t\right)=t^{n-h}\left(\sum_{i=1}^{l} t^{m_{i}}\right) \operatorname{Poin}(R, t) .
$$

Let $\Omega_{S}^{l-1}$ be the space of differential $l-1$ forms on $V$ with coefficients in $S$. Grade $\Omega_{S}^{l-1}$ in the natural way. Define the star-operator $*: \Omega_{S}^{1} \rightarrow \Omega_{S}^{l-1}$ by $*\left(f d x_{i}\right)=(-1)^{i-1} f d x_{1} \wedge \cdots \wedge d x_{i-1} \wedge d x_{i+1} \wedge \cdots \wedge d x_{l}$ [Fla, p.15, p.82]. Then $*(w \theta)=\operatorname{det}(w) w(* \theta)$ for $w \in W$. Since $*: \Omega_{S}^{1} \rightarrow \Omega_{S}^{l-1}$ is an isomorphism of graded $S$-modules, it follows that the restriction of $*$ to $\left(\Omega_{S}^{1}\right)^{\operatorname{det}}$ defines an isomorphism

$$
\left(\Omega_{S}^{1}\right)^{\operatorname{det}} \simeq\left(\Omega_{S}^{l-1}\right)^{W}
$$

of graded vector spaces. It is shown in [Sol1, Theorem] that $\left(\Omega_{S}^{l-1}\right)^{W}$ is a free $R$-module with basis $\psi_{i}=d f_{1} \wedge \cdots \wedge d f_{i-1} \wedge d f_{i+1} \wedge \cdots \wedge d f_{l}$ for $1 \leq i \leq l$. Define $\varphi_{i} \in \Omega_{S}^{1}$ by $* \varphi_{i}=\psi_{i}$. It follows that $\varphi_{1}, \ldots, \varphi_{l}$ is an $R$-module basis for $\left(\Omega_{S}^{1}\right)^{\operatorname{det}}$. Since $\operatorname{deg}\left(\varphi_{i}\right)=n-m_{i}$ we have

$$
\operatorname{Poin}\left(\left(\Omega_{S}^{1}\right)^{\operatorname{det}}, t\right)=\left(\sum_{i=1}^{l} t^{n-m_{i}}\right) \operatorname{Poin}(R, t) .
$$

Compare (6.5) and (6.7). By duality in the exponents we have $n-h+m_{i}=$ $n-m_{l-i+1}$ for $1 \leq i \leq l$. Thus $\operatorname{Poin}\left(\bigoplus_{i=1}^{l} R \hat{d} f_{i}, t\right)=\operatorname{Poin}\left(\left(\Omega_{S}^{1}\right)^{\operatorname{det}}, t\right)$. This completes the proof.

\section{Bibliography}

[Ath] C. Athanasiadis, On free deformations of the braid arrangement, European J. of Combinatorics 19 (1998), 7-18.

[Bou] N. Bourbaki, Groupes et Algèbres de Lie, Chapitres 4,5 et 6, Hermann, Paris 1968.

[EdR] P. Edelman and V. Reiner, Free arrangements and rhombic tilings, Discrete and Computational Geometry 15 (1996), 307-340.

[Fla] H. Flanders, Differential Forms, with Applications to the Physical Sciences, Academic Press, 1963.

[Mac] I. G. Macdonald, Symmetric functions and Hall polynomials, second ed., Oxford Univ. Press, 1995.

[OrT] P. Orlik and H. Terao, Arrangements of Hyperplanes. Grundlehren der Math. Wiss. 300, Springer Verlag, 1992.

[Sai1] K. Saito, On the uniformization of complements of discriminant loci. In: Conference Notes, Amer. Math. Soc. Summer Institute, Williamstown, 1975.

[Sai2] K. Saito, On a linear structure of a quotient variety by a finite reflexion group. RIMS Kyoto preprint 288, $1979=$ Publ. Res. Inst. Math. Sci. 29 (1993), 535-579. 
[Sai3] K. Saito, Theory of logarithmic differential forms and logarithmic vector fields, J. Fac. Sci. Univ. Tokyo Sect.IA Math. 27 (1980), 265-291.

[SYS] K. Saito, T. Yano and J. Sekiguchi, On a certain generator system of the ring of invariants of a finite reflection group, Communications in Algebra 8 (1980), 373-408.

[Shi1] J.-Y. Shi, The Kazhdan-Lusztig cells in certain affine Weyl groups, Lecture Notes in Math. 1179, Springer Verlag, 1986.

[Shi2] J.-Y. Shi, Sign types corresponding to an affine Weyl group, J. London Math. Soc. 35 (1987) 56-74.

[Sol1] L. Solomon, Invariants of finite reflection groups, Nagoya Math. J. 22 (1963), 57-64.

[Sol2] L. Solomon, Invariants of Euclidean reflection groups, Trans. Amer. Math. Soc. 113 (1964), 274-286.

[Sta1] R. Stanley, Hyperplane arrangements, interval orders, and trees, Proc. Nat. Acad. Sci. 93 (1996), 2620-2625.

[Sta2] R. Stanley, Hyperplane arrangements, parking functions, and tree inversions, preprint, 1996.

[Ter] H. Terao, Free arrangements of hyperplanes and unitary reflection groups, Proc. Japan Acad. Ser. A 56 (1980), 389-392.

[Zie] G. M. Ziegler, Multiarrangements of hyperplanes and their freeness, In: Singularities, Contemporary Math. 90, Amer. Math. Soc. (1989) 345-359.

Louis Solomon

Mathematics Department

University of Wisconsin

Madison WI 53706, USA
Hiroaki Terao

Mathematics Department

University of Wisconsin

Madison WI 53706, USA

e-mail: hterao@facstaff.wisc.edu

Current address:

Mathematics Department

Hokkaido University

Sapporo 060 JAPAN

e-mail: terao@math.sci.hokudai.ac.jp

(Received: March 13, 1997) 